\title{
Cylindricity Error Measuring and Evaluating for Engine Cylinder Bore in Manufacturing Procedure
}

\author{
Qiang Chen, ${ }^{1}$ Xueheng Tao, ${ }^{1,2}$ Jinshi Lu, ${ }^{1,2}$ and Xuejun Wang ${ }^{1,2}$ \\ ${ }^{1}$ Key Laboratory for Seafood Processing Technology and Equipment of Liaoning Province, Dalian Polytechnic University, \\ Dalian 116034, China \\ ${ }^{2}$ National Engineering Research Center of Seafood, Dalian 116034, China
}

Correspondence should be addressed to Xueheng Tao; xhtao@dlpu.edu.cn

Received 8 January 2016; Revised 29 February 2016; Accepted 2 March 2016

Academic Editor: Gianluca Percoco

Copyright (C) 2016 Qiang Chen et al. This is an open access article distributed under the Creative Commons Attribution License, which permits unrestricted use, distribution, and reproduction in any medium, provided the original work is properly cited.

\begin{abstract}
On-line measuring device of cylindricity error is designed based on two-point method error separation technique (EST), which can separate spindle rotation error from measuring error. According to the principle of measuring device, the mathematical model of the minimum zone method for cylindricity error evaluating is established. Optimized parameters of objective function decrease to four from six by assuming that $c$ is equal to zero and $h$ is equal to one. Initial values of optimized parameters are obtained from least square method and final values are acquired by the genetic algorithm. The ideal axis of cylinder is fitted in MATLAB. Compared to the error results of the least square method, the minimum circumscribed cylinder method, and the maximum inscribed cylinder method, the error result of the minimum zone method conforms to the theory of error evaluation. The results indicate that the method can meet the requirement of engine cylinder bore cylindricity error measuring and evaluating.
\end{abstract}

\section{Introduction}

The performance and quality of the engine are affected directly by the cylindricity error of the cylinder bore. How to accurately assess the cylindricity error has always been the focus of many researches. Murthy (1982) proposed the orthogonal polynomial method to evaluate the cylindricity error, which projects the measurement data of the cylindrical surface onto a plane, and the minimum zone solution is obtained by the method of calculating the flatness [1]. Carr and Ferreira (1995) studied the theory that the minimum zone problem can be converted into a series of linear programming problems [2]. Tian et al. (1999) put forward a method of minimum zone of cylindricity error based on computational geometry theory, the accurate location of minimum zone center is got by using the property of the convex hull of a set of nodes in a plane about Voronoi diagram, and then we will know the precise value of the cylindricity error [3]. Bei (2008) developed a precise model to evaluate the cylindricity error by adopting genetic algorithm [4].
The least square method is the most common method because it has the advantages of the mature theory, the specific mathematical formula, and easiness of implementation. When the ideal axis of cylinder is gaining, all the measuring points participate in the calculating procedure, which will lead to a large error. However, it is not suitable while error evaluating results are controversial. The minimum zone method is the only method that conforms to the definition of the cylindrical error in the national standard, and its results are used as the basis for the determination. The minimum zone method for evaluating cylindricity error is finding two coaxial cylindrical surfaces that can fully contain the actual cylinder bore cylindrical surface and make the radius difference minimize. This study, which focuses on the minimum zone method, posits a new method to evaluate the cylindricity error by genetic algorithm. The on-line measuring device of the cylindricity error is designed to achieve the coordinate data and the mathematical model is established according to the principle of the measuring device. Optimal parameters of ideal axis are obtained by genetic algorithm. The error result is compared to the results of three other methods, and it 


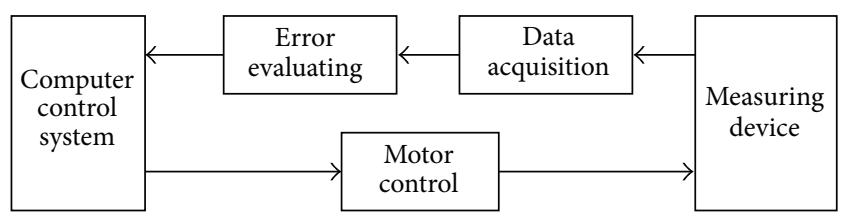

FIGURE 1: Structure of error measuring and evaluating.

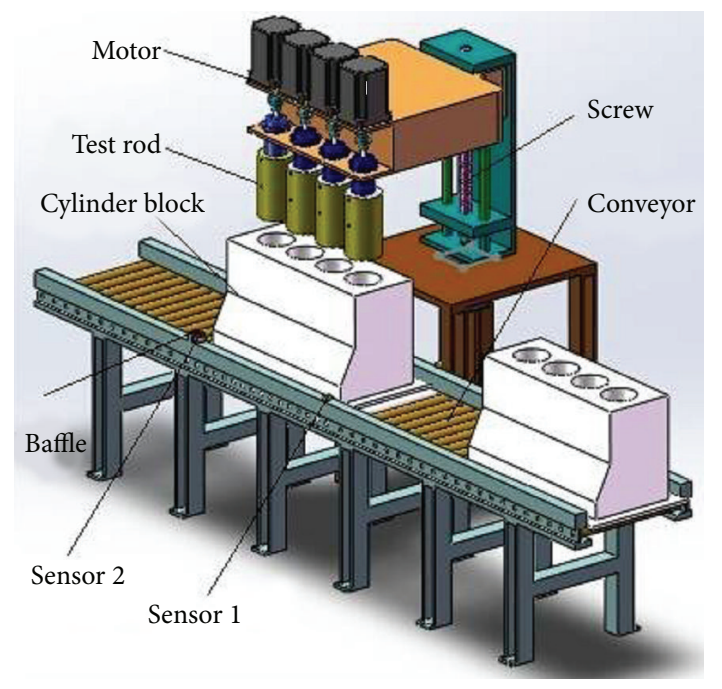

Figure 2: Cylindricity error measuring device.

indicates the minimum zone method, which adopted genetic algorithm, can reduce the error evaluation result effectively.

\section{Materials and Methods}

The structure of the system for error measuring and evaluating consists of measuring device, data acquisition, error evaluating, motor control, and computer control system. Figure 1 shows the structure of the system.

2.1. Cylindricity Error Measuring Device. The on-line measuring device of the cylindricity error is mainly composed of cylinder blocks, a conveyor, a positioning platform, two sensors, a baffle, a screw lifting platform, four stepper motors, and four test rods [5], as shown in Figure 2. The measured cylinder blocks move forward under the action of the conveyor. When sensor 1 detects a measured cylinder block, the baffle is stretched out, and when sensor 2 detects this measured cylinder block, the positioning platform (shown in Figure 3) is lifted up $5 \mathrm{~mm}$ in order to separate the measured cylinder block from the conveyor. Test rods that are driven by stepper motors move into the cylinder bore under the function of the screw lifting platform. Sensors on the measuring head read the data of different measuring points automatically and they are stored into the txt file for further processing. While the measuring process is finished, test rods depart from the measured cylinder bore and the positioning platform drops down $5 \mathrm{~mm}$, so as to make the measured

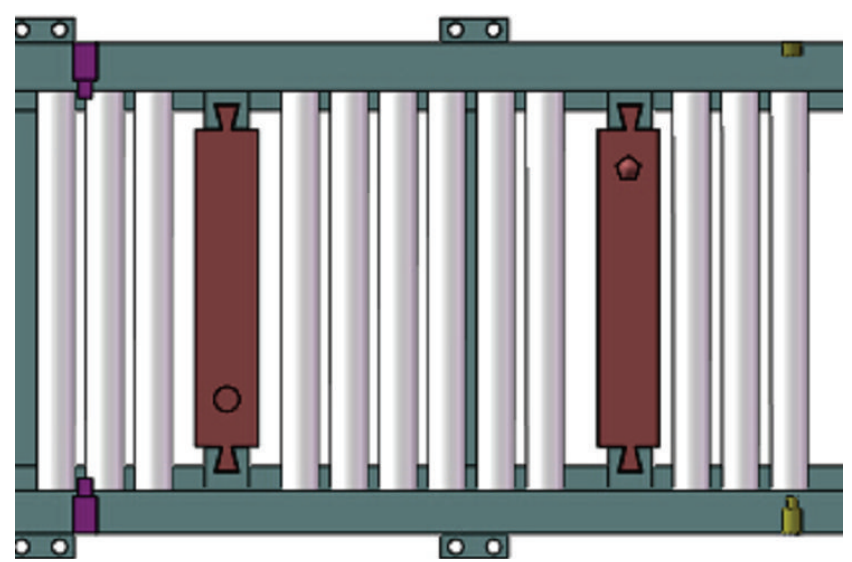

Figure 3: Positioning platform.

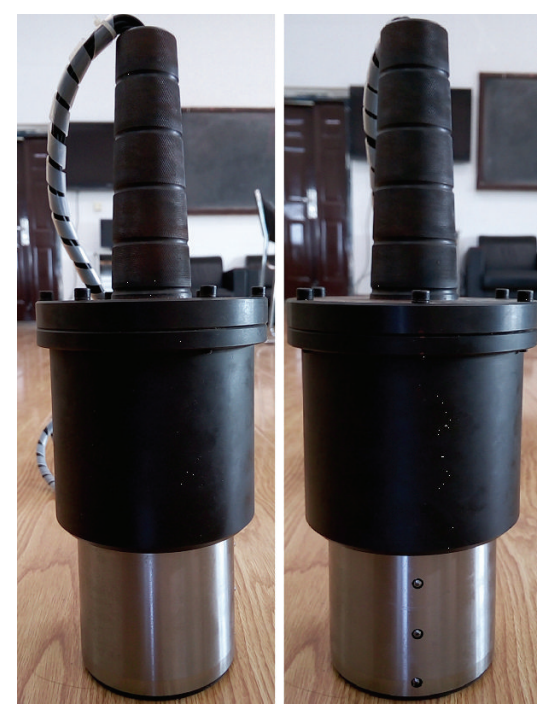

FIGURE 4: Test rod.

cylinder block touch the conveyor again. The next cylinder block measuring process will go on.

The multiprobe method can restrain the random error of the measurement system, which makes it possible to measure the workpiece without high precision rotation axis [6]. It is more suitable for on-line measuring and it plays a very important role in the measuring process for the large workpiece that cannot move. The two-point method of the error separation technique is adopted in this study, which performs at the aspect that one test rod has three layers and every two sensors that are arranged on each layer are separated by 180 degrees (opposite each other), as shown in Figure 4.

The equation of two-point method is as follows:

$$
\begin{aligned}
& u_{1}=r+h, \\
& u_{2}=-r+h,
\end{aligned}
$$

where $u_{1}$ and $u_{2}$ are the measuring error of sensors, $r$ is the roundness error, and $h$ is the spindle rotation error. 


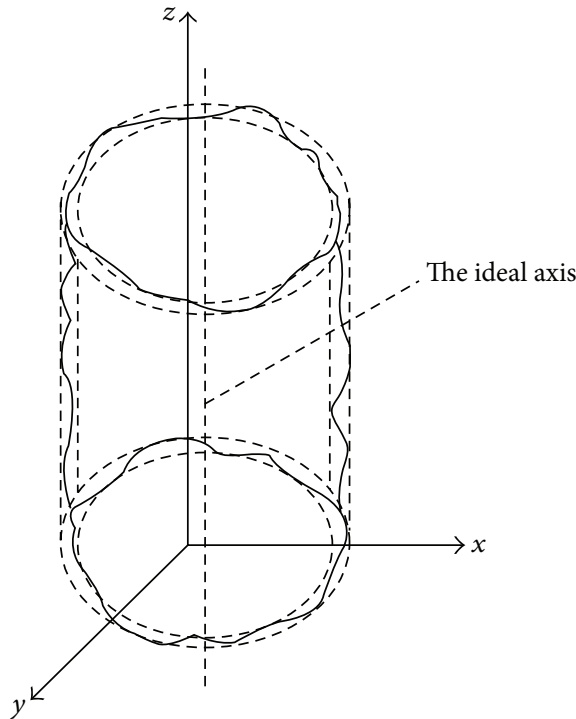

FIgURE 5: The minimum zone method.

The roundness error can be separated from the measuring error, and its value is as follows:

$$
r=\frac{\left(u_{1}-u_{2}\right)}{2}
$$

this will decrease the measuring error effectively and contribute to reduce the cylindricity error.

2.2. Mathematical Model. The evaluating process of the cylindricity error is the process of finding the ideal axis, also the process of approaching the ideal axis. When the approaching position and direction are different, the radius difference is not the same. The minimum value of the radius difference can be regarded as the cylindricity error, and the corresponding axis is the ideal axis. Figure 5 shows the minimum zone method of the cylindricity error.

The space rectangular coordinate system OXYZ is established by taking the rotary center of the test rod as the origin [7], and $m$ measured sections are selected equidistantly along the axis of the cylinder bore and $n$ measured points are selected equiangularly. The coordinate of the measuring point can be expressed as

$$
P_{i j}\left(r_{i j}, \theta_{i j}, z_{i j}\right), \quad i=1,2,3, \ldots, m, j=1,2,3, \ldots, n,
$$

where $j$ is the quantity of measured sections, $i$ is the quantity of measured points on every measured section, $r_{i j}$ is the real radius of the measured points to the axis, and $\theta_{i j}$ is the corresponding angle. If the ideal axis and a end face of the cylinder cross at the point $Q(a, b, c)$ and the direction ratio of the ideal axis is $(f, g, h)$, then the measured points can be written as follows:

$$
\begin{aligned}
& x_{i j}=r_{i j} \cos \theta_{i j}, \\
& y_{i j}=r_{i j} \sin \theta_{i j}, \\
& z_{i j}=z_{i j}
\end{aligned}
$$

so the equation of the ideal axis can be defined as [8]

$$
\frac{x_{i j}-a}{f}=\frac{y_{i j}-b}{g}=\frac{z_{i j}-c}{h}
$$

and the distance from the measuring points to the ideal axis can be expressed as

$$
R=\frac{\left|\begin{array}{ccc}
i & j & k \\
x_{i j}-a & y_{i j}-b & z_{i j}-c \\
f & g & h
\end{array}\right|}{\sqrt{f^{2}+g^{2}+h^{2}}} .
$$

As long as the optimal parameters' values about $a, b, c, f, g$, and $h$ are obtained, the equation of ideal axis is certain, and the cylindricity error is calculated. However, it is difficult to optimize since the formula is nonlinear [9]. In order to calculate conveniently, it is necessary to make some assumption: $h=1, c=0$; this will make optimal parameters reduce to four from six. The formula can simplify as

$$
R=\sqrt{\left(x_{i j}-f z_{i j}-a\right)^{2}+\left(y_{i j}-g z_{i j}-b\right)^{2}}
$$

and the objective function of cylindricity error can be defined as

$$
f(a, b, f, g)=\max \left\{R_{i}\right\}-\min \left\{R_{i}\right\}
$$

when the four optimal parameters are got, the cylindricity error can also be solved from formula (8).

\subsection{Error Evaluation}

2.3.1. Least Square Method for Initial Values. In order to reduce the optimization time, the least square method is used to obtain the initial values of four optimization parameters [10]. Equation (5) can also be written as follows:

$$
\begin{aligned}
& x=a+f z, \\
& y=b+g z .
\end{aligned}
$$

According to the principle of least square method that is given as

$$
Q=\sum_{i=1}^{n} \sum_{j=1}^{m} \varepsilon_{i j}^{2}=\min
$$

the objective function of least square method can be defined as

$$
f(a, b, f, g)=f_{\mathrm{LSC}}=\min \left\{\sum_{i=1}^{n} \sum_{j=1}^{m}\left(R_{i j}-R\right)^{2}\right\} .
$$


The initial values of parameters $a, b, f$, and $g$ can be calculated by the following formulas:

$$
\begin{aligned}
& a=\frac{\sum_{j=1}^{n} z_{j} \sum_{i=1}^{m} \sum_{j=1}^{n} x_{i j} z_{j}-\sum_{i=1}^{m} \sum_{j=1}^{n} x_{i j} \sum_{j=1}^{n} z_{j}^{2}}{m\left[\left(\sum_{j=1}^{n} z_{j}\right)^{2}-n \sum_{j=1}^{n} z_{j}^{2}\right]}, \\
& b=\frac{\sum_{j=1}^{n} z_{j} \sum_{i=1}^{m} \sum_{j=1}^{n} y_{i j} z_{j}-\sum_{i=1}^{m} \sum_{j=1}^{n} y_{i j} \sum_{j=1}^{n} z_{j}^{2}}{m\left[\left(\sum_{j=1}^{n} z_{j}\right)^{2}-n \sum_{j=1}^{n} z_{j}^{2}\right]}, \\
& f=\frac{\sum_{j=1}^{n} z_{j} \sum_{i=1}^{m} \sum_{j=1}^{n} x_{i j}-m \sum_{i=1}^{m} \sum_{j=1}^{n} x_{i j} z_{j}}{m\left[\left(\sum_{j=1}^{n} z_{j}\right)^{2}-n \sum_{j=1}^{n} z_{j}^{2}\right]} \\
& g=\frac{\sum_{j=1}^{n} z_{j} \sum_{i=1}^{m} \sum_{j=1}^{n} y_{i j}-m \sum_{i=1}^{m} \sum_{j=1}^{n} y_{i j} z_{j}}{m\left[\left(\sum_{j=1}^{n} z_{j}\right)^{2}-n \sum_{j=1}^{n} z_{j}^{2}\right]} .
\end{aligned}
$$

The iteration will begin from the initial values, and then the genetic algorithm will be used to obtain the final values.

2.3.2. Genetic Algorithm for Final Values. The genetic algorithm is a global and random search algorithm proposed by the American Professor J. Holland. Compared to the traditional optimization algorithms, it has the advantages of global search and can deal with nonlinear optimization [11]. So it is more suitable to be used to optimize the parameters of the objective function of the minimum zone method.

The main steps of the genetic algorithm are encoding, generating the initial population, selecting fitness function, searching, and outputting optimal parameters [12]. The process is showed in Figure 6.

Each parameter represents a gene and a chromosome is a solution. The expression $u=(a, b, f, g)$ is a chromosome composed of four parameters.

Fitness evaluation is the criterion of guiding the searching direction of the genetic algorithm, and only the fitness function is needed in the searching process. How to select an appropriate fitness function to evaluate the performance of the population is the key of the genetic algorithm. The fitness function is usually converted from the objective function [13]. In this study, the reciprocal of the objective function of the cylindricity error is chosen to be the fitness function, and the formula is given as

$$
\text { fit }(a, b, f, g)=\frac{1}{\max \left\{R_{i}\right\}-\min \left\{R_{i}\right\}} \text {. }
$$

The genetic searching mainly includes selection, crossover, mutation, and migration. Its purpose is to choose a better individual according to the principle of survival of the fittest. The best individual is the parameters' values.

The roulette wheel is taken as the selection operator, and the probability of the individual that is chosen to enter the next generation is given as

$$
P=\frac{\text { fit }\left(u_{k}\right)}{\sum_{k=1}^{N} \text { fit }\left(u_{k}\right)} .
$$

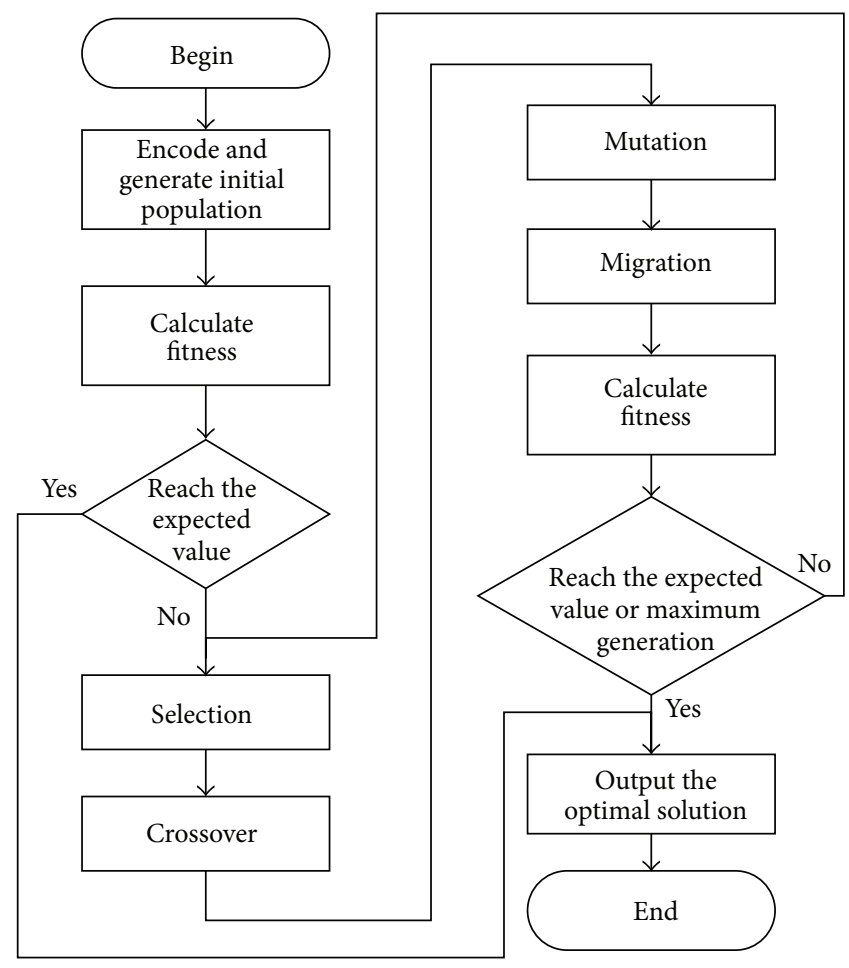

FIGURE 6: The process of the genetic algorithm.

The crossover operator plays an important role in the searching process. Its specific operation is setting a crosspoint randomly and exchanging the components of the two individuals, which will generate two new individuals. Two new individuals will be used for the next iteration. The crossover probability usually varies from 0.6 to 0.8 .

The mutation will generate new individuals by changing the gene on the chromosome. The fitness value of the new individual will be compared with the value of the original individual, and the mutation will continue until the latest individual is found and the mutation probability varies from 0.01 to 0.1 .

In order to solve the problem of local optimal solution, the transfer operator is introduced into the genetic algorithm. Some best individuals are migrated to other subgroups. When fitness reaches the expected value or the generation reaches maximum, the algorithm will be ended [14].

\section{Results and Discussion}

The cylinder block is tested by the on-line measuring device of the cylindricity error, and the rectangular coordinates data that is converted by the measuring coordinate data is showed in Tables 1, 2, and 3 .

In order to get the intuitive result, let us fit the measuring points in MATLAB, as shown in Figure 7.

Program the $m$-file of the fitness function, and enter the gatool in the command window of MATLAB; a genetic algorithm optimization toolbox will pop up. Set parameters as follows. 
TABLE 1: Measuring points on section $1(/ \mathrm{mm})$.

\begin{tabular}{lcc}
\hline$x$ & $y$ & $z$ \\
\hline 49.9793 & 0 & 30.0000 \\
43.2836 & 24.9896 & 30.0000 \\
24.9901 & 43.2841 & 30.0000 \\
0 & 49.9806 & 30.0000 \\
-24.9903 & 43.2852 & 30.0000 \\
-43.2852 & 24.9910 & 30.0000 \\
-49.9821 & 0 & 30.0000 \\
-43.2862 & -24.9911 & 30.0000 \\
-24.9914 & -43.2853 & 30.0000 \\
0 & -49.9808 & 30.0000 \\
24.9901 & -43.2841 & 30.0000 \\
43.2835 & -24.9898 & 30.0000 \\
\hline
\end{tabular}

TABLE 2: Measuring points on section 2 (/mm).

\begin{tabular}{lcc}
\hline$x$ & $y$ & $z$ \\
\hline 49.9796 & 0 & 60.0000 \\
43.2836 & 24.9894 & 60.0000 \\
24.9902 & 43.2840 & 60.0000 \\
0 & 49.9808 & 60.0000 \\
-24.9904 & 43.2851 & 60.0000 \\
-43.2753 & 24.9909 & 60.0000 \\
-49.9819 & 0 & 60.0000 \\
-43.2858 & -24.9911 & 60.0000 \\
-24.9809 & -43.2852 & 60.0000 \\
0 & -49.9809 & 60.0000 \\
24.9903 & -43.2844 & 60.0000 \\
43.2834 & -24.9896 & 60.0000 \\
\hline
\end{tabular}

TABLE 3: Measuring points on section 3 (/mm).

\begin{tabular}{lcc}
\hline$x$ & $y$ & $z$ \\
\hline 49.9798 & 0 & 90.0000 \\
43.2797 & 24.9894 & 90.0000 \\
24.9902 & 43.2841 & 90.0000 \\
0 & 49.9808 & 90.0000 \\
-24.9904 & 43.2851 & 90.0000 \\
-43.2849 & 24.9908 & 90.0000 \\
-49.9817 & 0 & 90.0000 \\
-43.2859 & -24.9909 & 90.0000 \\
-24.9810 & -43.2853 & 90.0000 \\
0 & -49.9814 & 90.0000 \\
24.9899 & -43.2845 & 90.0000 \\
43.2831 & -24.9893 & 90.0000 \\
\hline
\end{tabular}

The population size is 20 , the selection operator is the roulette wheel, the crossover operator is the single-point crossover, the crossover probability is 0.8 , the mutation probability is 0.1 , the migration interval is 20 , the migration probability is 0.2 , and the number of the iteration is 100 .

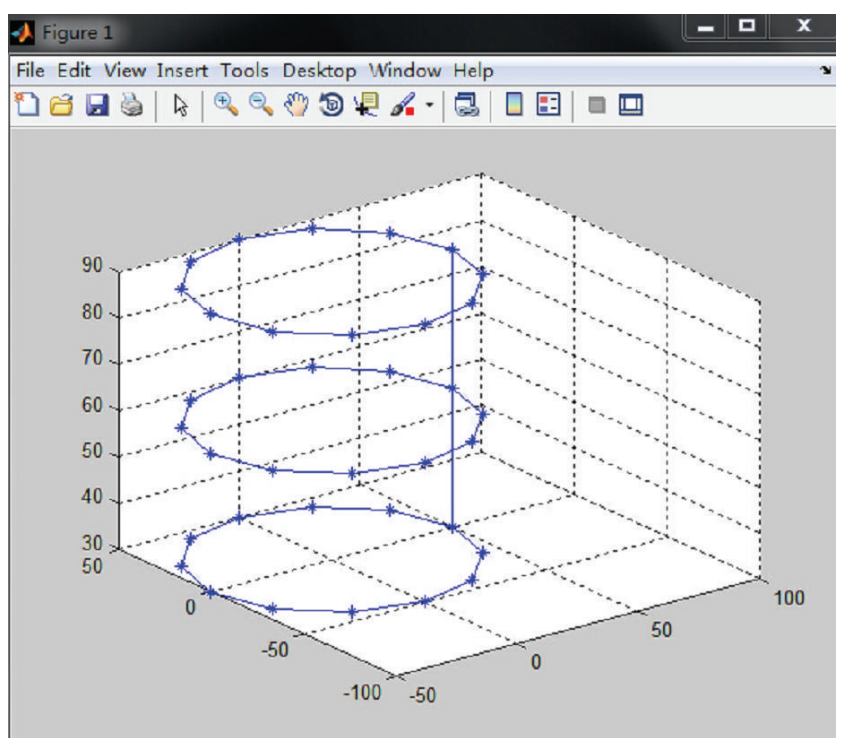

FIGURE 7: The measuring points fitting.

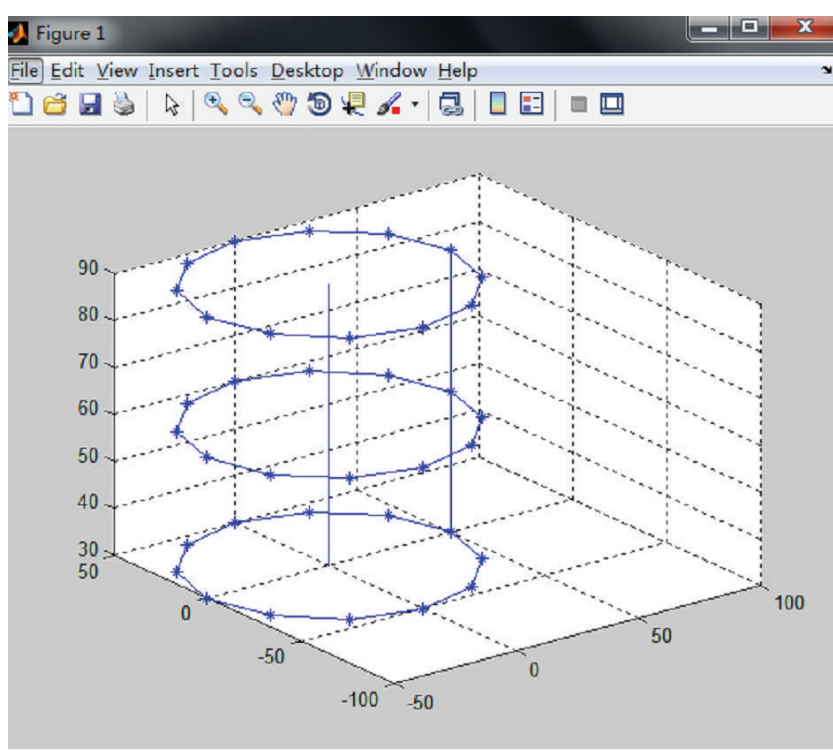

Figure 8: The ideal axis.

The iteration is terminated after 52 generations and the optimal parameters of $a, b, f$, and $g$ are obtained by the minimum zone method and the final parameters' values are $a=-0.3709, b=-0.0064, f=0.0033$, and $g=0.0063$. The equation of the ideal axis (5) is expressed as follows:

$$
\frac{x+0.3709}{0.0033}=\frac{y+0.0064}{0.0063}=z .
$$

The ideal axis is obtained, as shown in Figure 8.

We can get the 3D cylinder of the measuring data, and it shows the different roundness from the different position in Figure 9. Figure 10 is one of the sections, and the roundness error can be obtained from this section. 


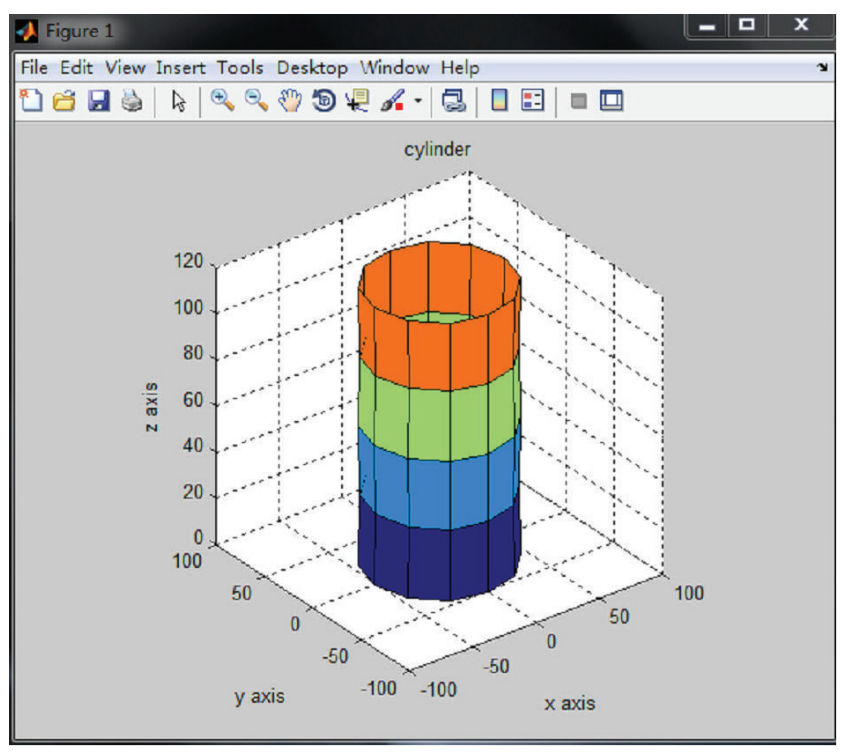

Figure 9: The 3D cylinder of measuring points.

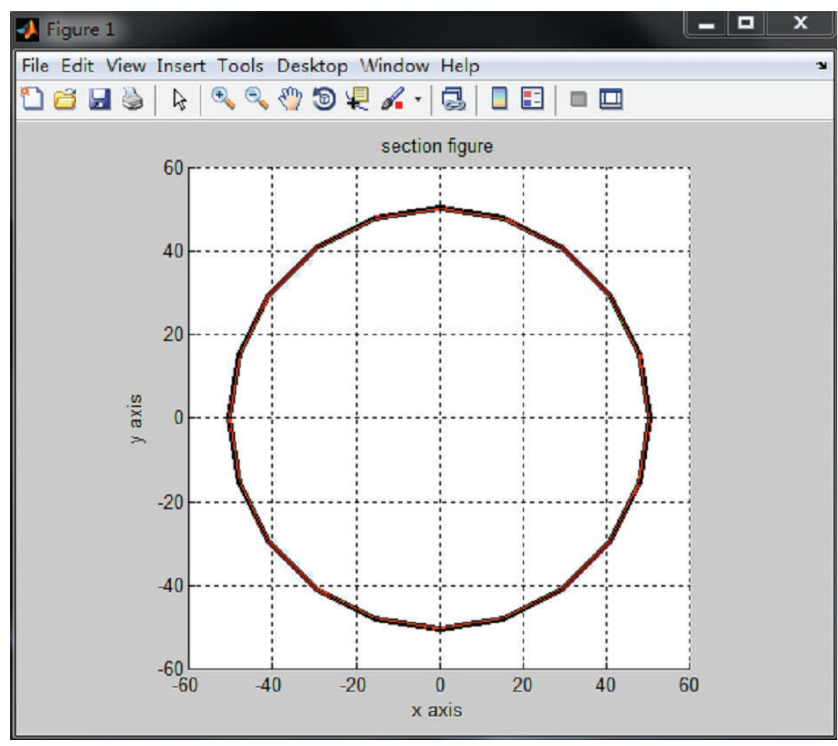

Figure 10: The one section of cylinder.

The cylindricity error evaluation system (shown in Figure 11) is finished in the MFC of VC++ 6.0, and the final error value can be calculated as $0.008 \mathrm{~mm}$ in this system.

In order to verify the accuracy of the algorithm, the least square method, the minimum circumscribed cylinder method, and the maximum inscribed cylinder method are adopted to compare the results of the minimum zone method. Table 4 shows their results.

We can get the message that the result of minimum zone method is minimum, and it can meet the requirement of cylindricity error evaluating for engine cylinder block.
TABLE 4: The cylindricity error of four methods (/mm).

\begin{tabular}{lccccc}
\hline & $a$ & $b$ & $p$ & $q$ & Error \\
\hline MZC & -0.3709 & -0.0064 & 0.0033 & 0.0063 & 0.008 \\
LSC & 0.2731 & 0.0061 & -0.0035 & -0.0041 & 0.012 \\
MCC & 0.2640 & 0.0069 & 0.0019 & -0.0036 & 0.015 \\
MIC & -0.2713 & 0.0053 & 0.0022 & -0.0045 & 0.013 \\
\hline
\end{tabular}

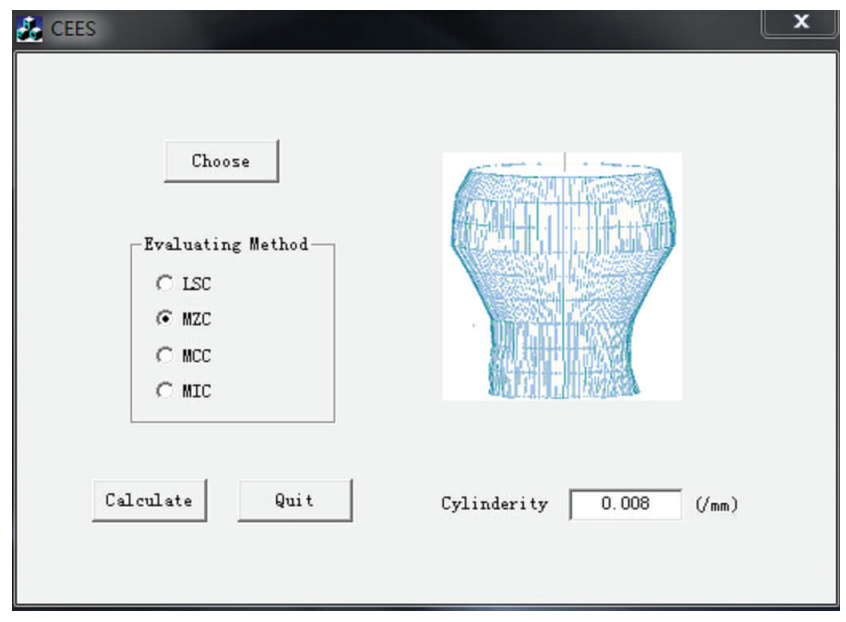

FIgURE 11: The error evaluating system.

\section{Conclusion}

In this paper, an effective measuring device of cylindricity error has been designed. The two-point method error separation technique adopted on the test rods can reduce spindle rotary error. The mathematical model for cylindricity error evaluating is constructed according to the principle of measuring device and the optimized parameters of ideal axis are reduced to four from six. The initial value is got by least square method and final value is achieved by genetic algorithm. The ideal axis is fitting in MATLAB and the error evaluated value of minimum zone method is compared to the value of least square method, which shows that the method can achieve a better result for cylindricity error measuring and evaluating.

In future study, it is necessary to comprehensively analyze the factors, which affect the final accuracy of cylindricity error measuring and evaluating. Further research on error evaluating will be discussed in the future.

\section{Competing Interests}

The authors declare that there are no competing interests regarding the publication of this paper.

\section{Acknowledgments}

The authors acknowledge the National Engineering Research Center of Seafood. This work was supported by the Public 
Science and Technology Research Funds Projects of Ocean (no. 201505029).

\section{References}

[1] T. S. R. Murthy, "A comparison of different algorithms for cylindricity evaluation," International Journal of Machine Tool Design and Research, vol. 22, no. 4, pp. 283-292, 1982.

[2] K. Carr and P. Ferreira, "Verification of form tolerances part II: cylindricity and straightness of a median line," Precision Engineering, vol. 17, no. 2, pp. 144-156, 1995.

[3] G. Y. Tian, Z. X. Zhao, and R. W. Baines, "The design of miniaturized displacement transducers for deep hole diameter measurement," Mechatronics, vol. 9, no. 7, pp. 317-327, 1999.

[4] G. X. Bei, "Evaluation method of cylindricity error based on genetic algorithm," Journal of Shandong University (Engineering Science), vol. 38, no. 2, pp. 34-36, 2008.

[5] L. Zhang, Y. Zhao, and L. Ba, "Research and practice on the online measurement of cylindricity error in a grinding machine," Key Engineering Materials, vol. 87, no. 359, pp. 176-180, 2008.

[6] Y. Q. Wei, "Some mathematical models of measurement of cylindricity error with multi-point method," Journal of China Textile University, no. 2, pp. 34-39, 1996.

[7] X. Zhang, X. Jiang, and P. J. Scott, "A reliable method of minimum zone evaluation of cylindricity and conicity from coordinate measurement data," Precision Engineering, vol. 35, no. 3, pp. 484-489, 2011.

[8] P. Liu and H. Miao, "Mathematical model for evaluating cylindricity errors by minimum zone method," Advanced Materials Research, vol. 284-286, pp. 434-438, 2011.

[9] N. Venkaiah and M. S. Shunmugam, "Evaluation of form data using computational geometric techniques-part II: cylindricity error," International Journal of Machine Tools and Manufacture, vol. 47, no. 7-8, pp. 1237-1245, 2007.

[10] X. Q. Lei, H. W. Song, Y. J. Xue, J. Li, J. Zhou, and M. Duan, "Method for cylindricity error evaluation using Geometry Optimization Searching Algorithm," Measurement, vol. 44, no. 9, pp. 1556-1563, 2011.

[11] J. A. Niesse and H. R. Mayne, "Global geometry optimization of atomic clusters using a modified genetic algorithm in spacefixed coordinates," The Journal of Chemical Physics, vol. 105, no. 11, pp. 4700-4706, 1996.

[12] H.-Y. Lai, W.-Y. Jywe, C.-K. Chen, and C.-H. Liu, "Precision modeling of form errors for cylindricity evaluation using genetic algorithms," Precision Engineering, vol. 24, no. 4, pp. 310-319, 2000.

[13] J.-B. Zhou and T. Sun, "Robustness and precision evaluation of the form error of micro-structured surfaces using real coded genetic algorithm," Journal of Beijing Institute of Technology (English Edition), vol. 21, no. 4, pp. 479-486, 2012.

[14] C. Chang, "Form error evaluation of circles based on a finelydesigned genetic algorithm," Chinese Journal of Mechanical Engineering, vol. 17, no. 1, pp. 59-62, 2004. 

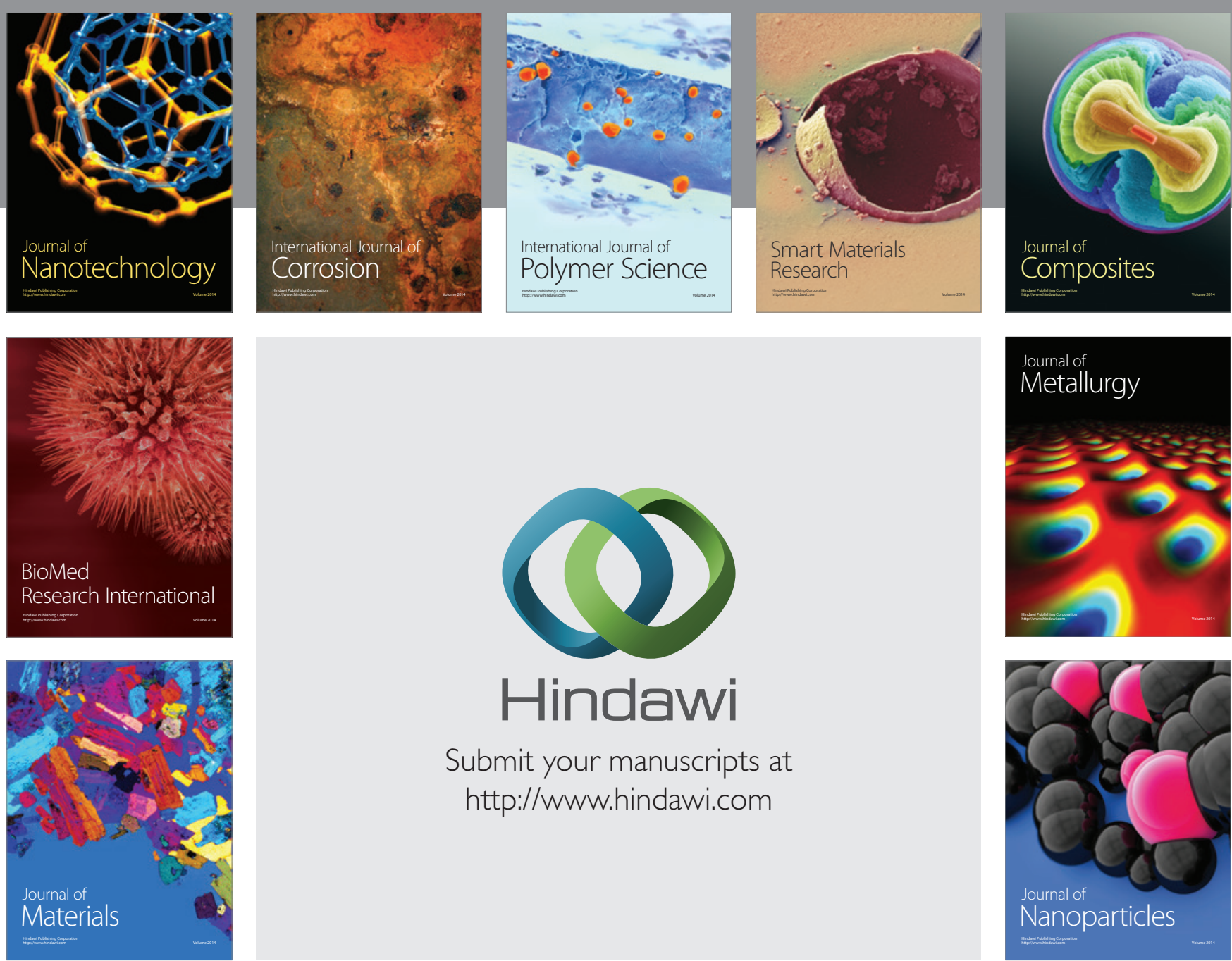

\section{Hindawi}

Submit your manuscripts at

http://www.hindawi.com

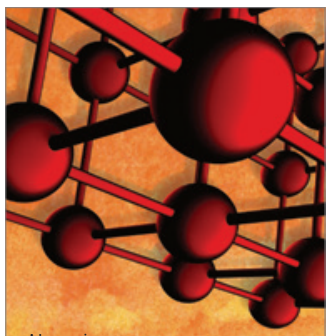

Materials Science and Engineering
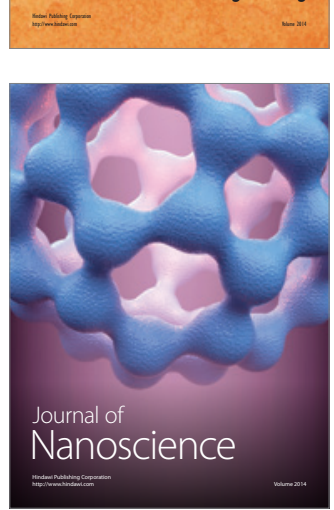
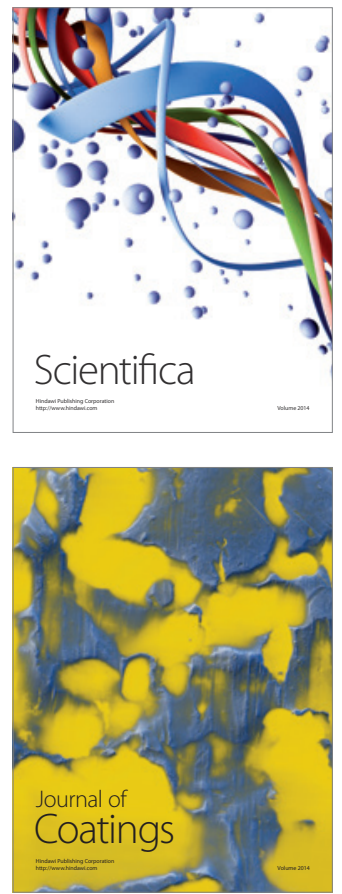
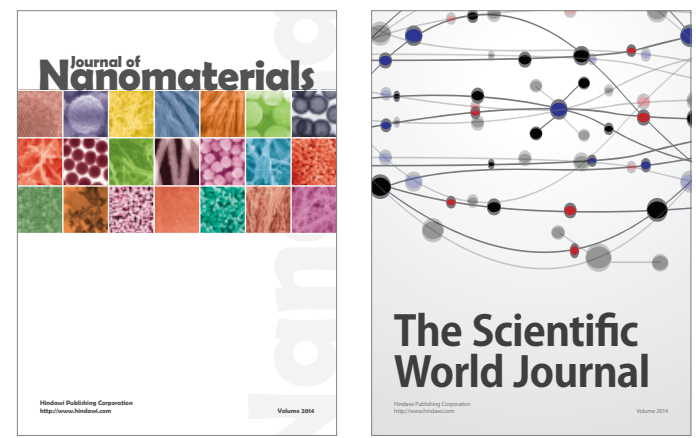

The Scientific World Journal
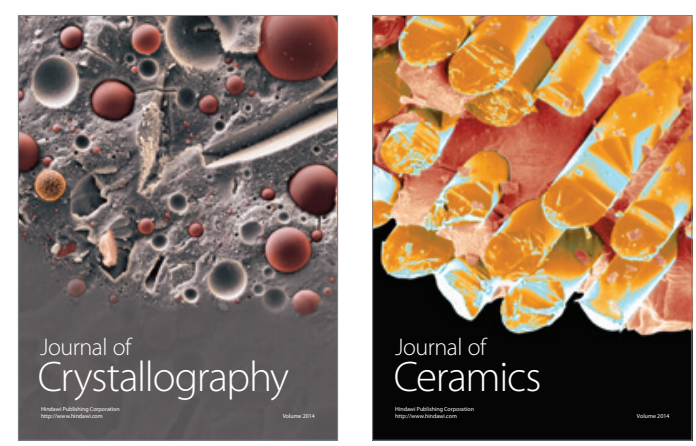
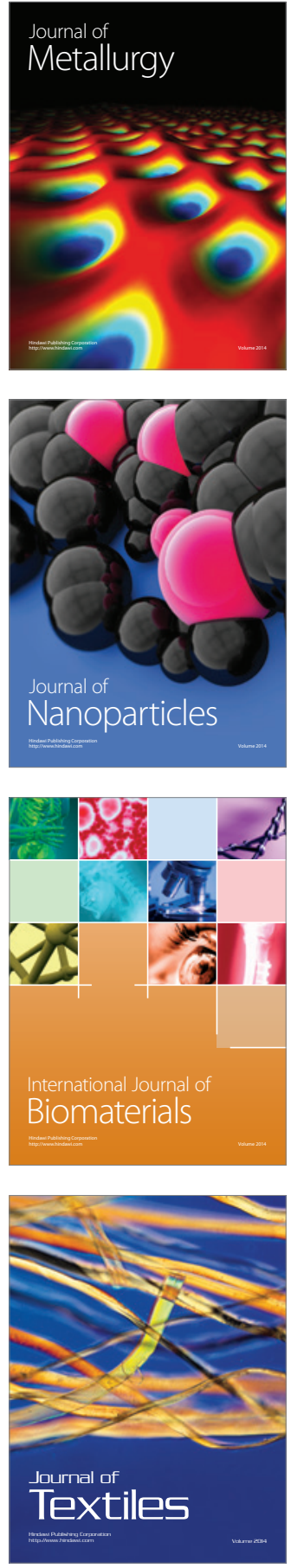